\title{
The Projective Character Tables of a Solvable Group $2^{6}:(6 \times 2)$
}

\author{
Abraham Love Prins $\mathbb{D}$ \\ Department of Mathematics and Physics, Faculty of Applied Sciences, Cape Peninsula University of Technology, P.O. Box 1906, \\ Bellville 7535, South Africa \\ Correspondence should be addressed to Abraham Love Prins; prinsab@cput.ac.za
}

Received 7 June 2019; Accepted 17 September 2019; Published 27 November 2019

Academic Editor: Adolfo Ballester-Bolinches

Copyright (c) 2019 Abraham Love Prins. This is an open access article distributed under the Creative Commons Attribution License, which permits unrestricted use, distribution, and reproduction in any medium, provided the original work is properly cited.

\begin{abstract}
The Chevalley-Dickson simple group $G_{2}(4)$ of Lie type $G_{2}$ over the Galois field GF(4) and of order $251596800=2^{12} \cdot 3^{3} \cdot 5^{2} \cdot 7 \cdot 13$ has a class of maximal subgroups of the form $2^{4+6}:\left(A_{5} \times 3\right)$, where $2^{4+6}$ is a special 2-group with center $Z\left(2^{4+6}\right)=2^{4}$. Since $2^{4}$ is normal in $2^{4+6}:\left(A_{5} \times 3\right)$, the group $2^{4+6}:\left(A_{5} \times 3\right)$ can be constructed as a nonsplit extension group of the form $\bar{G}=2^{4} \cdot\left(2^{6}:\left(A_{5} \times 3\right)\right)$. Two inertia factor groups, $H_{1}=2^{6}:\left(A_{5} \times 3\right)$ and $H_{2}=2^{6}:(6 \times 2)$, are obtained if $\bar{G}$ acts on $2^{4}$. In this paper, the author presents a method to compute all projective character tables of $H_{2}$. These tables become very useful if one wants to construct the ordinary character table of $\bar{G}$ by means of Fischer-Clifford theory. The method presented here is very effective to compute the irreducible projective character tables of a finite soluble group of manageable size.
\end{abstract}

\section{Introduction}

The Chevalley-Dickson simple group $G_{2}(4)$ of Lie type $G_{2}$ over the Galois field GF(4) and of order $251596800=$ $2^{12} \cdot 3^{3} \cdot 5^{2} \cdot 7 \cdot 13$ has exactly eight conjugacy classes of maximal subgroups [1]. One of these classes of maximal subgroups of index 1365 in $G_{2}(4)$ is a 2-local subgroup of the form $2^{4+6}:\left(A_{5} \times 3\right)$, where $2^{4+6}$ is a special 2-group with center $Z\left(2^{4+6}\right)=2^{4}$. Also, it can be mentioned that $2^{4+6}:\left(A_{5} \times 3\right)$ is a parabolic subgroup of $G_{2}(4)$.

The group $2^{4+6}:\left(A_{5} \times 3\right)$ is the normalizer $N_{G_{2}(4)}\left((2 A)^{4}\right)[1]$ of an elementary abelian 2-group $(2 A)^{4}$ of order 16 in $G_{2}(4)$, where the generators of $(2 A)^{4}$ are 4 commuting involutions found in the class of involutions $2 \mathrm{~A}$ of $G_{2}(4)$. Hence, we can construct $2^{4+6}:\left(A_{5} \times 3\right)$ as an extension group of $N=2^{4}$ by $G=2^{6}:\left(A_{5} \times 3\right)$. Using a permutation representation of degree 416 of $2^{4+6}:\left(A_{5} \times\right.$ 3 ) found in [2], we can easily verify with the help of the computer algebra system GAP [3] that $2^{4+6}:\left(A_{5} \times 3\right)$ exists as a nonsplit extension group $\bar{G}=2^{4 \cdot}\left(2^{6}:\left(A_{5} \times 3\right)\right)$. In fact, from the character table of $2^{4+6}:\left(A_{5} \times 3\right)$ uploaded in the GAP library, we can deduce that $2^{6}$ is also normal in
$2^{4+6}:\left(A_{5} \times 3\right)$. Therefore, with the aid of GAP, it can be easily shown that the group $2^{4+6}:\left(A_{5} \times 3\right)$ is also isomorphic to a nonsplit extension of the form $2^{6 \cdot}\left(2^{4}\right.$ : $\left.\left(A_{5} \times 3\right)\right)$.

Since $N_{G_{2}(4)}\left((2 A)^{4}\right) \cong \bar{G}$, the action of $G=2^{6}:\left(A_{5} \times 3\right)$ on $N=2^{4}$ splits $N$ into two orbits of lengths 1 and 15. By Brauer's theorem in [4], $G$ also acts on $\operatorname{Irr}(N)$ with two orbits of lengths 1 and 15 and the corresponding inertia factor subgroups are of the forms $H_{1}=G$ and $H_{2}=2^{6}:(6 \times 2)$. If one wants to construct the ordinary character table of $\bar{G}$ by the technique of Fischer-Clifford matrices [5], the irreducible ordinary characters $\operatorname{Irr}\left(H_{1}\right)$ of $H_{1}$ and either the ordinary irreducible characters $\operatorname{Irr}\left(H_{2}\right)$ or a set of irreducible projective characters $\operatorname{IrProj}\left(\mathrm{H}_{2}, \alpha^{-1}\right)$ of $\mathrm{H}_{2}$ with associated nontrivial factor set $\alpha^{-1}$ are needed. Readers are referred to [6] on a survey of Fischer-Clifford theory.

From the uploaded character table in the GAP library, we found that $\bar{G}$ has 42 conjugacy classes. Hence, by Fischer-Clifford theory, $|\operatorname{Irr}(\bar{G})|=|\operatorname{Irr}(G)|+\mid \operatorname{Irr} \operatorname{Proj}\left(H_{2}\right.$, $\left.\alpha^{-1}\right) \mid=42$. Therefore, $\left|\operatorname{IrrProj}\left(H_{2}, \alpha^{-1}\right)\right|=16$, where $\mid \operatorname{Irr}$ $(G) \mid=26$. Since $\left|\operatorname{Irr}\left(H_{2}\right)\right|=30$, certainly a set of 
irreducible projective characters with nontrivial factor set $\alpha^{-1}$ of $\mathrm{H}_{2}$ is required in the construction of $\operatorname{Irr}(\bar{G})$ via Fischer-Clifford theory.

Using computations in GAP, the Schur multiplier $M$ $\left(\mathrm{H}_{2}\right) \cong 2^{4}$ of $\mathrm{H}_{2}$ is identified as a group isomorphic to the elementary abelian 2-group $2^{4}$. Therefore, the group $\mathrm{H}_{2}$ can have up to 15 sets of distinct irreducible projective characters $\operatorname{IrrProj}\left(H_{2}, \alpha_{i}^{-1}\right), i=2,3,4, \ldots, 16$, such that $\alpha^{2} \sim 1$. In this paper, the author presents a method (based on the work done in $[7,8])$ to compute all the distinct irreducible projective character tables of $\mathrm{H}_{2}$. The author will also show how to choose the appropriate set $\operatorname{IrrProj}\left(\mathrm{H}_{2}, \alpha^{-1}\right)$ for $\mathrm{H}_{2}$ if one wants to construct the set $\operatorname{Irr}(\bar{G})$ using the technique of Fischer-Clifford matrices.

A proof is given of a result, which states that the number of irreducible projective characters |IrrProj $\left(G, \alpha^{-1}\right) \mid$ of a finite group $G$ associated with some factor set $\alpha^{-1}$ is always less or equal to the number of the ordinary irreducible characters $|\operatorname{Irr}(G)|$ of $G$. Based on the abovementioned proof, GAP codes are developed to find the number $\left|\operatorname{IrrProj}\left(\mathrm{H}_{2}, \alpha_{i}^{-1}\right)\right|$ of irreducible projective characters of $\mathrm{H}_{2}$ associated with each factor set $\alpha_{i}^{-1}$ in a cohomology class $\left[\alpha_{i}\right]$ of $M\left(H_{2}\right)$. Interested readers are referred to [9-13] for definitions on concepts in ordinary and projective character theory. Computations are carried out with the aid of the computer algebra systems MAGMA [14] and GAP [3], and the notation of ATLAS [1] is mostly used.

\section{Preliminary Results on Projective Characters}

In this section, a brief overview of relevant projective character theory pertaining to our study is given. $G$ will always denote a finite group. Also, a proof of a proposition is given which states that the number of irreducible projective characters of a finite group $G$ associated with some factor set $\alpha$ is always less or equal to the number of the ordinary irreducible characters of $G$.

Definition 1. A function $\alpha: G \times G \longrightarrow \mathbb{C}^{*}$ is called a factor set of $G$ if $\alpha(x y, z) \alpha(x, y)=\alpha(x, y z) \alpha(y, z)$ for all $x, y, z \in G$.

The set of all equivalence classes of factor sets of $G$ forms a finite abelian group, called the Schur multiplier, and is denoted by $M(G)$.

Definition 2. A projective representation of a group $G$ of degree $n$ over the complex numbers is a map $P: G \longrightarrow \mathrm{GL}(n, \mathbb{C})$, such that

(i) $P(1)=I_{n}$

(ii) Given $x, y \in G$, there exists $\alpha(x, y) \in \mathbb{C}^{*}$ such that $P(x) P(y)=\alpha(x, y) P(x y)$

The map $\alpha$ is called the factor set associated with $P$.

Let $P$ be a projective representation of $G$ with factor set $\alpha$. Define $\kappa(g)=\operatorname{Trace}(P(g))$ for all $g \in G$. Then, $\kappa$ is called a projective character of $G$. We say that $\kappa$ is irreducible if $P$ is, and $\kappa$ has a factor set $\alpha$, where $\alpha$ is the factor set of $P$.
Let $\operatorname{IrrProj}(G, \alpha)$ denote the set of irreducible projective characters of $G$ associated with the factor set $\alpha$. An element $x \in G$ is said to be $\alpha$-regular if $\alpha(x, g)=\alpha(g, x)$ for all $g \in C_{G}(x)$. It is well known that $g \in G$ is $\alpha$-regular if and only if $\kappa(g) \neq 0$ for some $\kappa \in \operatorname{IrrProj}(G, \alpha)$ or equivalently that $g$ is $\alpha$-irregular if and only if $\kappa(g)=0$ for all $\kappa \in \operatorname{IrrProj}(G, \alpha)$. The number of irreducible projective characters with factor set $\alpha$ equals the number of $\alpha$-regular classes of a group $G$. Projective characters also satisfy the usual orthogonality relations and have analogues to ordinary characters.

As we will see later in Section 4, Definition 3 and Remark 1 will play an important role in the computation of the irreducible projective characters of $\mathrm{H}_{2}$.

Definition 3. A group $R$ is a representation group for $G$ if there exists a homomorphism $\pi$ from $R$ onto $G$ such that (i) $A=\operatorname{ker}(\pi) \cong M(G)$ and (ii) $A \leq Z(R) \cap R^{\prime}$.

Remark 1. A covering group $C$ for $G$ will normally be a quotient of $R$ by a subgroup $B$ of $A$. If $A / B$ has order $n$, we sometimes refer to the covering group as a $n$-fold cover of $G$. Projective representations of $G$ are found in the representation group $R$ for all the equivalence classes of factor sets in $M(G)$. However, in an $n$-fold cover $C$ of $G$, only the $n$ equivalence classes which $C$ covers will be represented [7].

The following proposition (see $[15,16]$ ) is useful to determine the number of irreducible projective characters of a group $G$ associated with a certain factor set $\alpha$. It tells us also under which condition $|\operatorname{IrrProj}(G, \alpha)|$ is strictly less than $|\operatorname{Irr}(G)|$. In Section 4, GAP codes (based on Proposition 1) will be used to compute the number $\left|\operatorname{IrrProj}\left(G, \alpha_{i}^{-1}\right)\right|$ of all irreducible projective characters of $\mathrm{H}_{2}$ found in a set $\operatorname{IrrProj}\left(G, \alpha_{i}^{-1}\right)$.

Proposition 1. Let $R=M(G)$. $G$ be a representation group of a finite group $G$, where $M(G)$ denotes the Schur multiplier of $G$. Then, the number of irreducible characters $\operatorname{Irr}(R)$ of $R$ which lie over a linear character $\theta \in \operatorname{Irr}(M(G))$ is less or equal to $|\operatorname{Irr}(G)|$.

Proof. The number of irreducible characters $\operatorname{Irr}(R)$ of $R$ which lies over a linear character $\theta \in \operatorname{Irr} M(G)$ is given by $\sum_{\chi \in \operatorname{Irr}(R)}<\chi \downarrow_{M(G)}, \theta>/ \chi(1)$. It is known that the quantity $\sum_{\chi \in \operatorname{Irr}(R)} \chi(x) / \chi(1) \geq 0$ for each $x \in M(G)$, and it is nonzero if $x$ is a commutator in $R$. For any $\theta \in \operatorname{Irr}(M(G))$, we have

$$
\begin{aligned}
\sum_{x \in M(G)} \sum_{\chi \in \operatorname{Irr}(R)} \frac{\chi(x) \theta\left(x^{-1}\right)}{\chi(1)} & \leq \sum_{x \in M(G)} \sum_{\chi \in \operatorname{Irr}(R)} \frac{\chi(x)}{\chi(1)} \\
& =|M(G)|\left|[g]_{R / M(G)}\right|
\end{aligned}
$$

where $\left|[g]_{R / M(G)}\right|$ is the number of conjugacy classes of $R / M(G) \cong G$. The last equality follows because the irreducible characters of $R$ with $M(G)$ in their kernels are precisely those which contain the trivial character on the restriction to $M(G)$. Hence, $(1 /|M(G)|) \sum_{x \in M(G)} \sum_{\chi \in \operatorname{Irr}(R)} \chi$ $(x) \theta\left(x^{-1}\right) / \chi(1)=\sum_{\chi \in \operatorname{Irr}(R)}(1 /|M(G)|) \sum_{x \in M(G)} \chi(x) \theta\left(x^{-1}\right) /$ 
TABle 1: Action of $G$ on $N$ and $\operatorname{Irr}(N)$.

\begin{tabular}{lcc}
\hline & Action of $G$ on $N$ & Action of $G$ on Irr $(N)$ \\
\hline Number and size of orbits $Q_{i}$ & $\left|O_{1}\right|=1$ & $\left|O_{1}\right|=1$ \\
\hline Structure of stabilizers $P_{i}$ and $H_{i}$ & $\left|O_{2}\right|=15$ & $\left|O_{2}\right|=15$ \\
\hline \multirow{2}{*}{ Size of stabilizers $P_{i}$ and $H_{i}$} & $P_{1}=2^{6}:\left(A_{5} \times 3\right)$ & $H_{1}=2^{6}:\left(A_{5} \times 3\right)$ \\
$P_{2}=2^{6}:(6 \times 2)$ & $\mid(6 \times 2)$ \\
\hline \multirow{2}{*}{ Number of conjugacy classes $[\mathrm{g}]$ of $P_{i}$ and $H_{i}$} & $\left|P_{1}\right|=11520$ & $\left|H_{1}\right|=11520$ \\
& $\left|P_{2}\right|=768$ & $\left|H_{2}\right|=768$ \\
\hline
\end{tabular}

$\chi(1)=\sum_{\chi \in \operatorname{Irr}(R)}<\chi \downarrow_{M(G)}, \theta>/ \chi(1) \leq\left|[g]_{R / M(G)}\right|=|\operatorname{Irr}(G)|$. Furthermore, if there is a nonidentity element $x \in M(G) \backslash \operatorname{ker}(\theta)$ which is a commutator in $R$, then the inequality becomes strict.

\section{Action of $2^{6}:\left(A_{5} \times 3\right)$ on $2^{4}$ and $\operatorname{Irr}\left(2^{4}\right)$}

As explained in the introductory section, the split extension $2^{4+6}:\left(A_{5} \times 3\right)$ can be constructed as a nonsplit extension group $\bar{G}=2^{4 \cdot}\left(2^{6}:\left(A_{5} \times 3\right)\right)$ of an elementary abelian group $N=2^{4}$ by $G=2^{6}:\left(A_{5} \times 3\right)$. Since $\bar{G}$ is the normalizer of $N$ in $G_{2}(4)$, the action of $\bar{G}$ on $N$ gives rise to two orbits of lengths 1 and 15. It follows that the action of $G$ on $N$ will also result in two orbits of lengths 1 and 15 with corresponding point stabilizers $P_{1}=G$ and $P_{2}=2^{6}:(6 \times 2)$. Then, by Brauer's theorem [4], $G$ also has two orbits of lengths 1 and 15 on $\operatorname{Irr}(N)$ with corresponding inertia factor subgroups $H_{1}=G$ and $H_{2}=2^{6}:(6 \times 2)$. See Table 1 for a summary of the action (which is self-dual) of $2^{6}:\left(A_{5} \times 3\right)$ on $2^{4}$ and $\operatorname{Irr}\left(2^{4}\right)$, respectively.

\section{Projective Character Tables of Inertia Factor $\mathrm{H}_{2}$}

In this section, all the sets $\operatorname{IrrProj}\left(\mathrm{H}_{2}, \alpha_{i}^{-1}\right)$ of irreducible projective characters of $H_{2}$ with associated factor sets $\alpha_{i}^{-1}$ will be computed. Appropriate GAP codes will be used to assist in the computational aspects of determining the sets $\operatorname{IrrProj}\left(\mathrm{H}_{2}, \alpha_{i}^{-1}\right)$. Readers are referred to $[7,8]$ for a background on the computational techniques being used in this section.

Since the Schur multiplier of $M\left(H_{2}\right) \cong 2^{4}$ is isomorphic to the elementary abelian group $2^{4}$ of order 16 , we obtain that $M\left(\mathrm{H}_{2}\right)$ contains 15 cohomology classes $\left[\alpha_{i}\right]$ of order 2 and the trivial class [1]. Hence, there exist 15 sets of projective characters $\operatorname{IrrProj}\left(\mathrm{H}_{2}, \alpha_{i}^{-1}\right)$ lying above the nontrivial factor sets $\alpha_{i}^{-1} \in\left[\alpha_{i}\right], i=2,3,4, \ldots, 16$, such that $\alpha_{i}^{2} \sim 1$. Note that the order $\left|H_{2}\right|=2^{8} .3$ of $H_{2}$ is the product of powers of two primes, and hence, by the Burnside's $p^{a} q^{b}$ theorem [17], the group $\mathrm{H}_{2}$ is solvable. Since $\mathrm{H}_{2}$ is of relative small order and it is solvable, the following GAP codes [18] (based on Proposition 1) give the number of irreducible projective characters of $\mathrm{H}_{2}$ contained in each set $\operatorname{IrrProj}\left(H_{2}, \alpha_{i}^{-1}\right)$ :

$$
\begin{aligned}
& \text { gap }>h:=H_{2} \\
& \text { gap }>f:=\text { EpimorphismSchurCover }(h)
\end{aligned}
$$

gap $>f:=$ InverseGeneralMapping(Isomorphism $\operatorname{PcGroup}(\operatorname{Source}(f))) * f$

gap $>z:=\operatorname{Kernel}(f)$

gap $>x:=\operatorname{Source}(f)$

gap $>\operatorname{List}(\operatorname{Irr}(z)$, lambda $->\operatorname{Number}(\operatorname{Irr}(x)$, chi $->$ not IsZero(ScalarProduct(RestrictedClassFunction(chi,z), lambda))))

$[30,18,26,14,16,4,16,4,16,4,16,4,16,4,16,4]$

From the output of the above GAP codes, the number of irreducible projective characters $\left|\operatorname{Irr} \operatorname{Proj}\left(\mathrm{H}_{2}, \alpha_{i}^{-1}\right)\right|$ of $\mathrm{H}_{2}$ contained in each set $\operatorname{IrrProj}\left(H_{2}, \alpha_{i}^{-1}\right)$ is $30,26,18,16,16,16$, $16,16,16,14,4,4,4,4,4$, and 4 , respectively. The set containing the 30 projective characters is associated with the trivial factor set $\alpha_{1}^{-1}=1$ of $M(G)$, and hence, they are the ordinary irreducible characters $\operatorname{Irr}\left(\mathrm{H}_{2}\right)$ of $\mathrm{H}_{2}$. Note in the above GAP codes that "Source $(f)$ " is the full representation group $R=M(G) \cdot H_{2}$ of $H_{2}$ whereas "Kernel $(f)$ " denotes the Schur multiplier $M(G)$.

From Remark 1, the irreducible projective characters of $H_{2}$ with a given factor set $\alpha_{i}^{-1}$ can be computed without using the full representation group $\mathrm{R}=\mathrm{M}\left(\mathrm{H}_{2}\right) \cdot \mathrm{H}_{2}$ of $\mathrm{H}_{2}$. Since all the nontrivial factor sets $\alpha_{i}^{-1}$ of $\operatorname{IrProj}\left(H_{2}, \alpha_{i}^{-1}\right)$ are of order two, the aim is to find 15 double covers $C_{i}$ of $\mathrm{H}_{2}$ which contain the desired 15 sets $\operatorname{IrrProj}\left(\mathrm{H}_{2}, \alpha_{i}^{-1}\right)$ of projective characters of $\mathrm{H}_{2}$. The nontrivial maximal subgroups $M_{i}$ of $M\left(\mathrm{H}_{2}\right)$ are computed within GAP, and we found there are exactly 15 of them of order 8 and shape $2^{3}$. The 15 factor groups $C_{i}=R / M_{i}, i=2,3,4, \ldots, 16$, are the double covers $2_{i} \cdot H_{2}$ which contain the 15 sets $\operatorname{IrrProj}\left(H_{2}, \alpha_{i}^{-1}\right)$ of desired irreducible projective characters of $\mathrm{H}_{2}$ with factor sets $\alpha_{i}^{-1}$ such that $\alpha_{i}^{2} \sim 1$. The following GAP code was used to "restrict" $R$ to $H_{2}$ to obtain the sets $\operatorname{IrrProj}\left(H_{2}, \alpha_{i}^{-1}\right)$, $i=2,3,4, \ldots, 16$ :

$$
\begin{aligned}
& \text { gap }>t:=\text { CharacterTable }\left(\text { “ } H_{2}\right. \text { ”) } \\
& \text { gap }>2 t:=\text { CharacterTable( }\left(C_{i}\right. \text { ”) } \\
& \text { gap }>F:=\operatorname{GetFusionMap}(2 t, t) \\
& \text { gap }>\text { map }:=\operatorname{ProjectionMap}(F) \\
& \text { gap }>\text { projchars }:=\operatorname{List}(2 t, x->\{x \operatorname{map}\})
\end{aligned}
$$

From the sets $\operatorname{IrrProj}\left(H_{2}, \alpha_{i}^{-1}\right)$ which contain 16 irreducible projective characters each, we obtain exactly 3 distinct irreducible projective character tables. Also, the sets $\operatorname{IrrProj}\left(H_{2}, \alpha_{i}^{-1}\right)$ which contain 4 irreducible projective characters each, give rise to 3 distinct sets of irreducible 


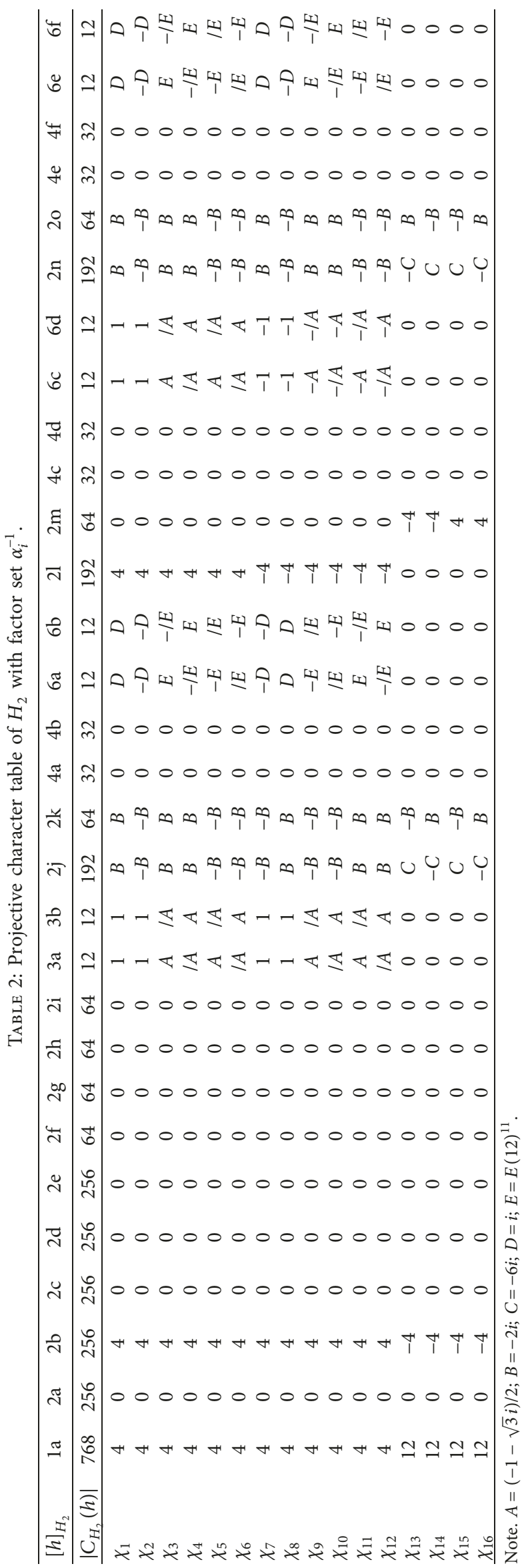




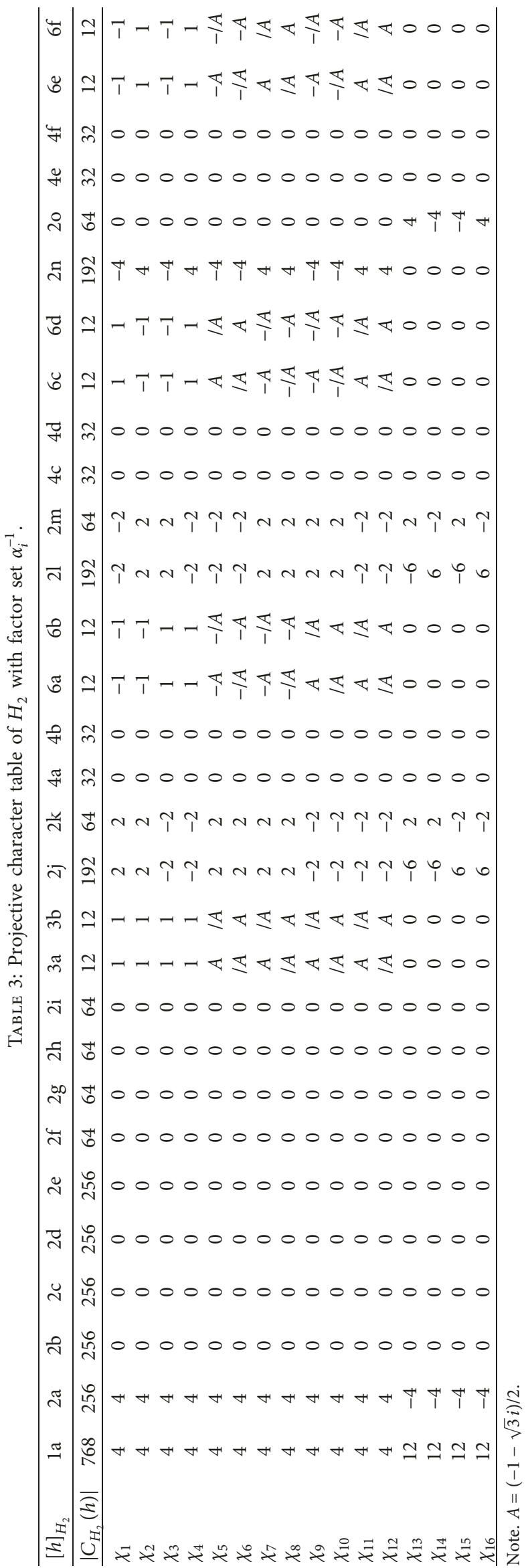




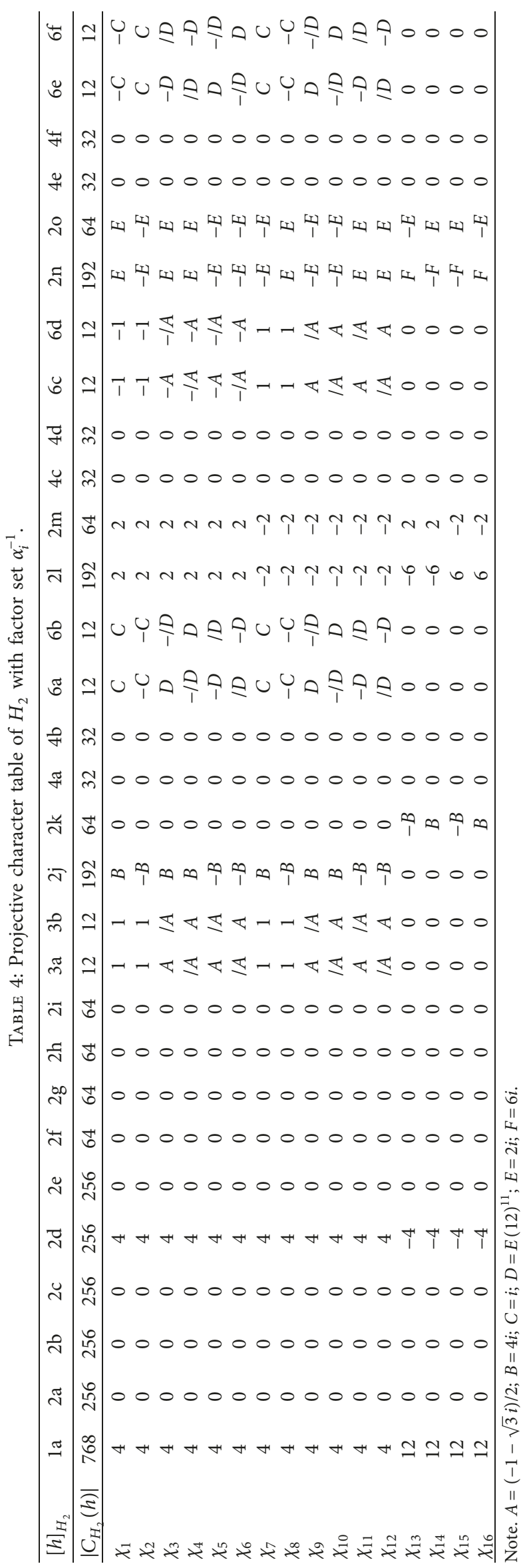




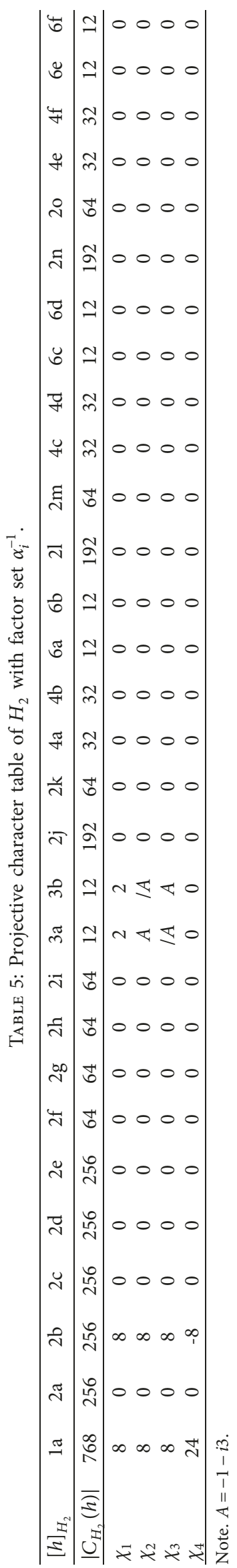




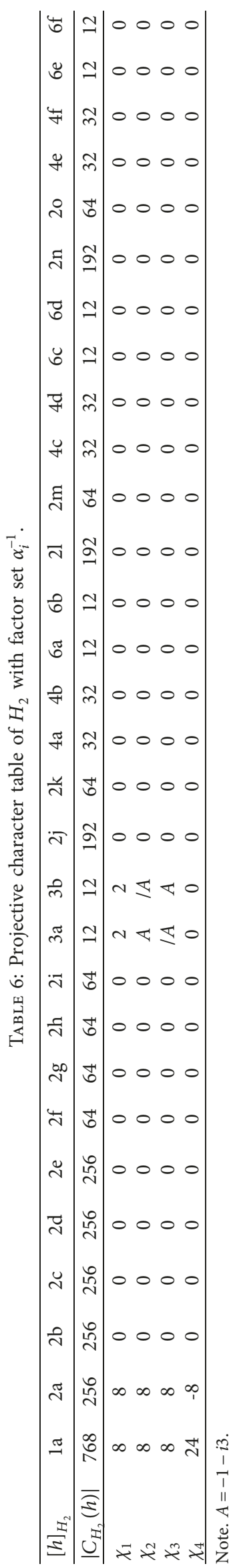




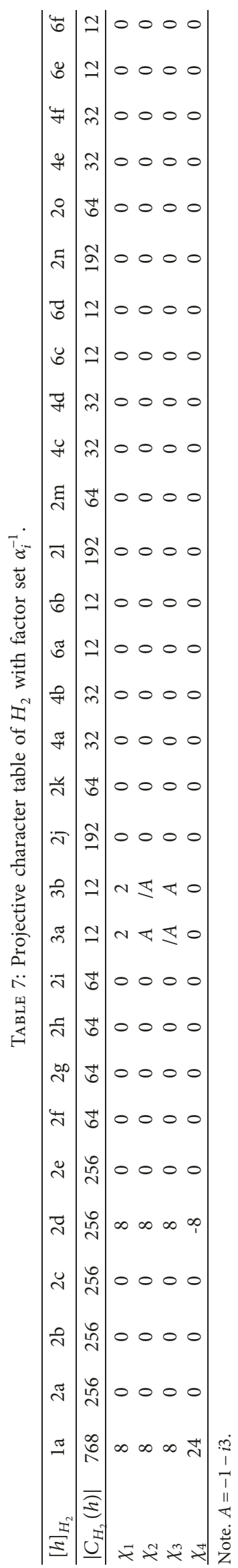




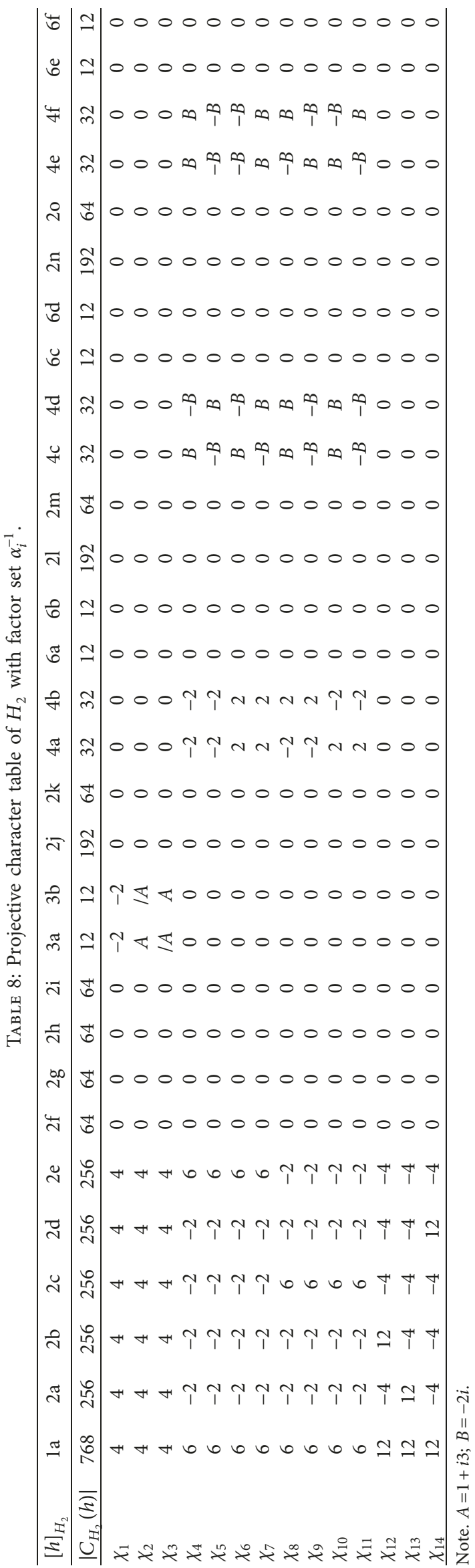




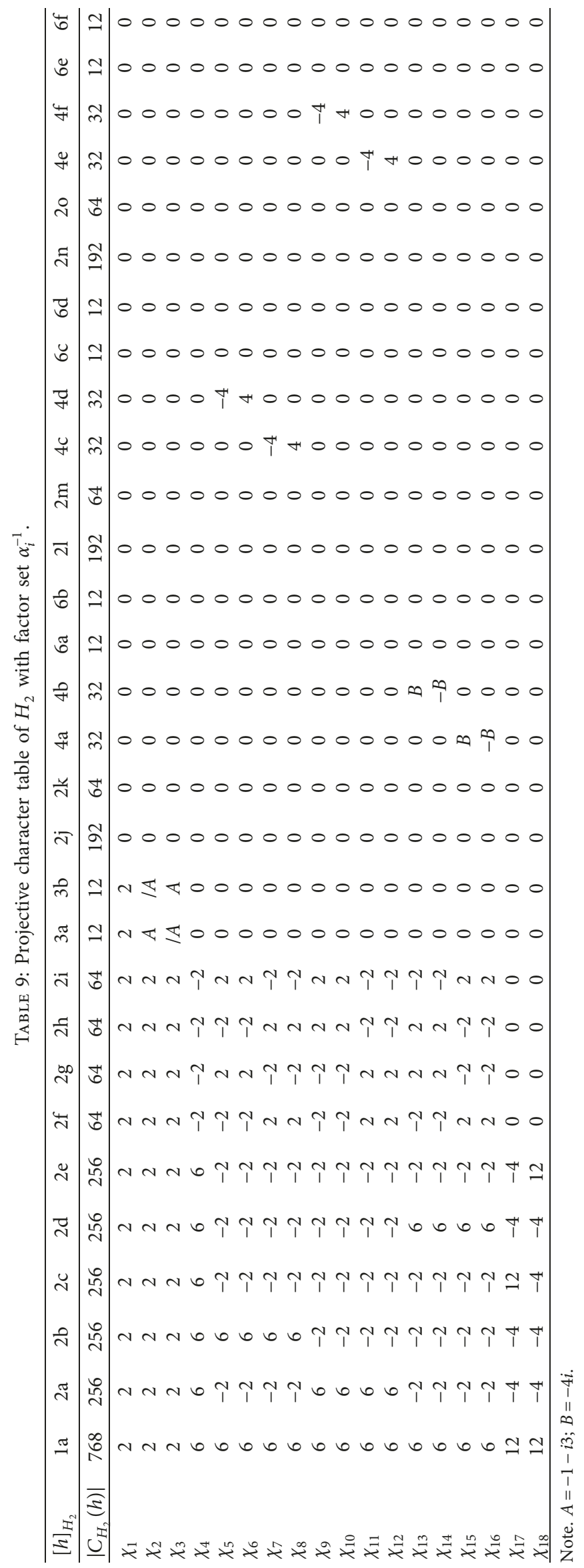




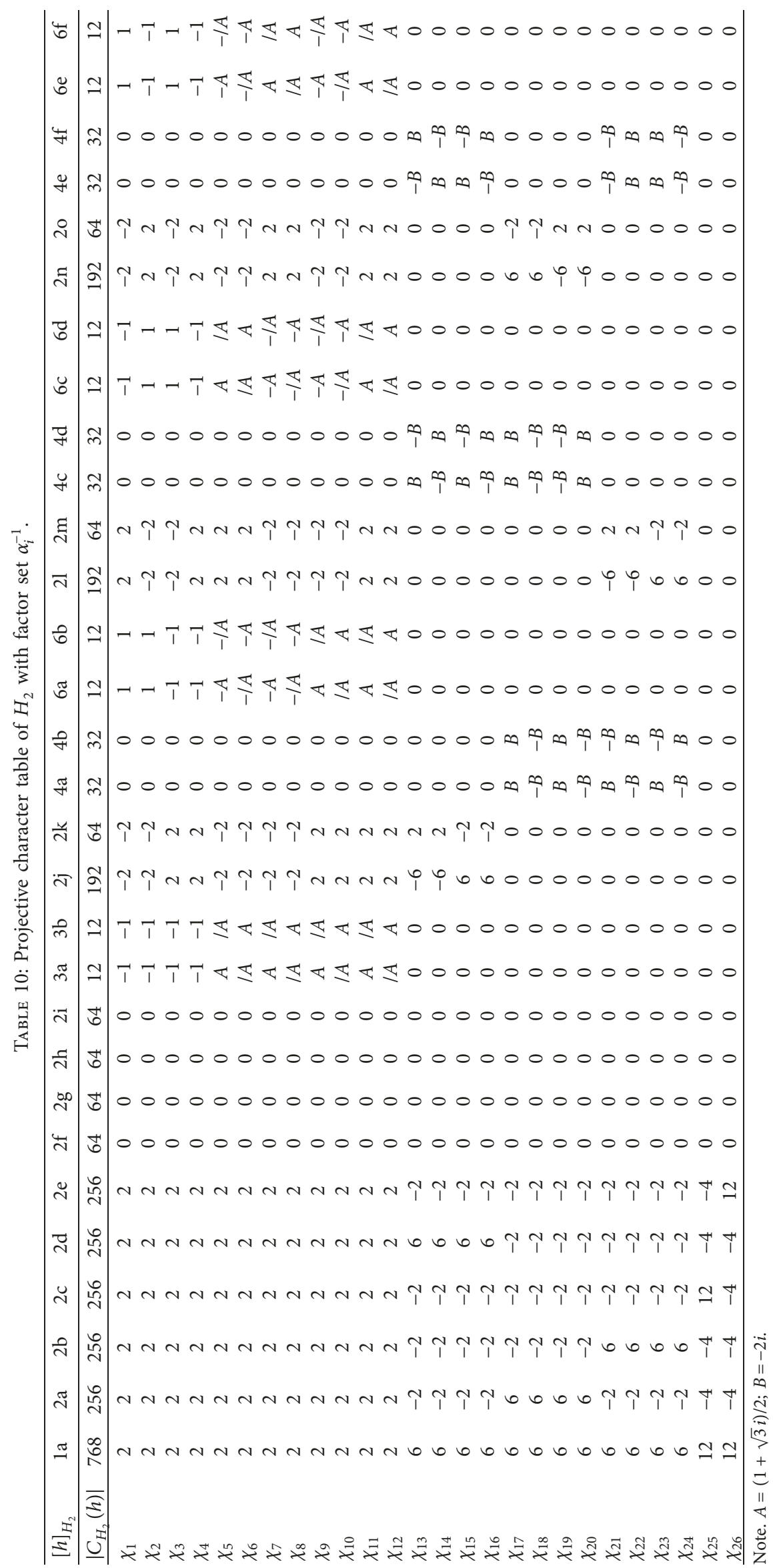




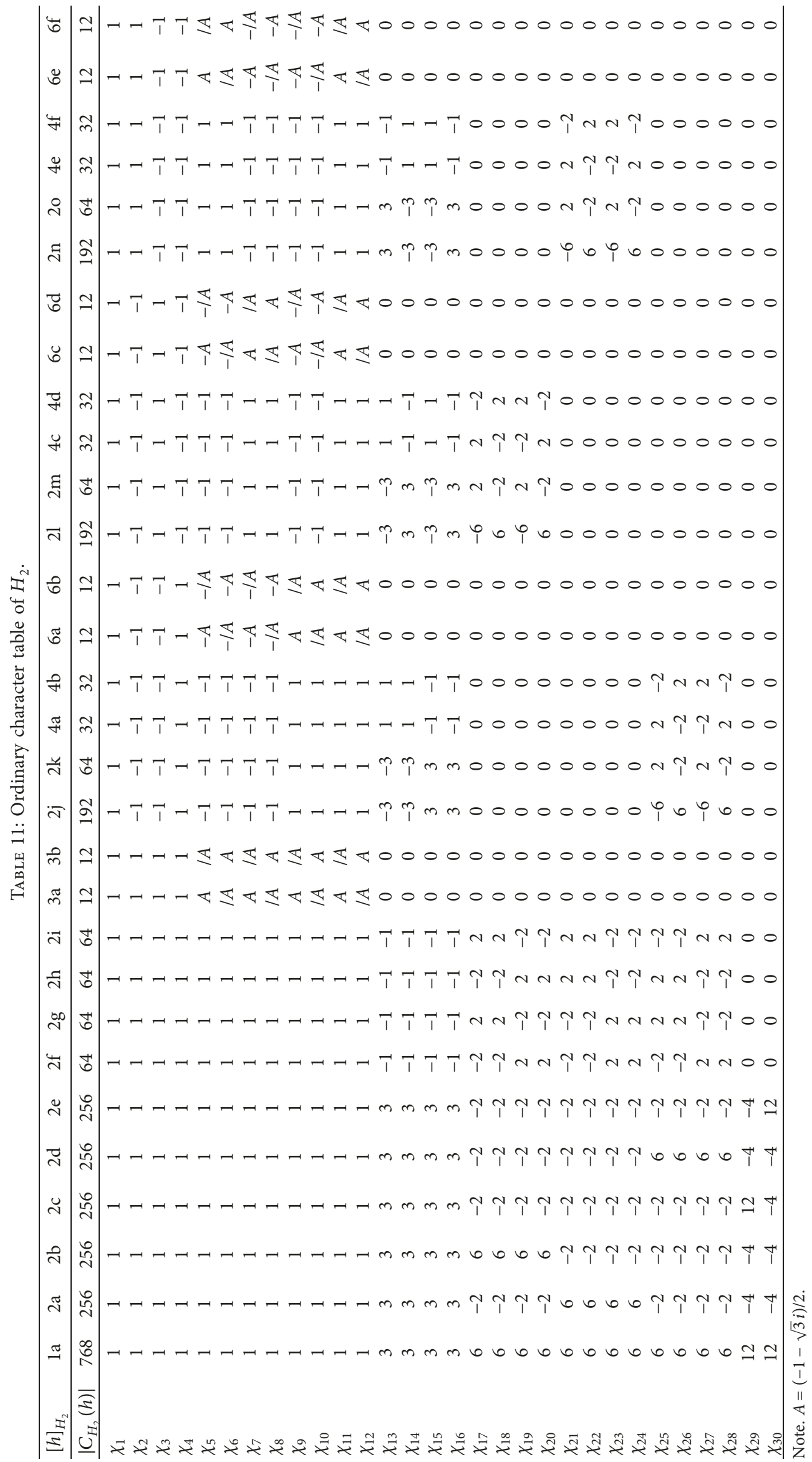


TABLE 12: Fischer-Clifford matrices of $\bar{G}$.

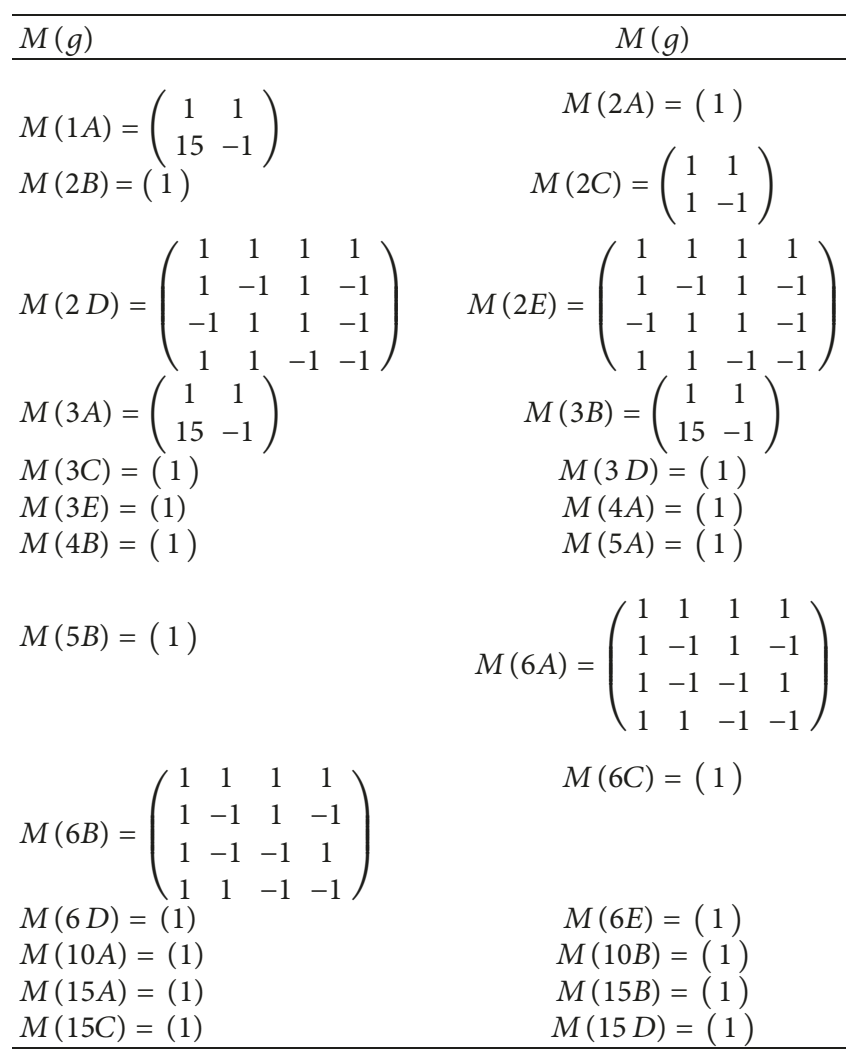

projective character tables. Hence, we obtain 9 distinct sets $\operatorname{IrrProj}\left(\mathrm{H}_{2}, \alpha_{i}^{-1}\right)$ of irreducible projective characters for $\mathrm{H}_{2}$, and they are listed in Tables 2-10. The ordinary irreducible characters $\operatorname{Irr}\left(\mathrm{H}_{2}\right)$ of $\mathrm{H}_{2}$ are found in Table 11. Note that the irreducible projective characters with associated factor set $\alpha_{i}^{-1}$ have values of zero on the $\alpha_{i}^{-1}$-irregular classes of $G$. Hence, the number of irreducible projective characters equals the number of $\alpha_{i}^{-1}$-regular classes of $G$ in each of the below tables. The above results are summarized in the following theorem:

Theorem 1. The inertia factor subgroup $\mathrm{H}_{2}$ of the action of $G$ on $\operatorname{Irr}(\mathrm{N})$ has exactly 9 distinct sets of $\operatorname{IrrProj}\left(\mathrm{H}_{2}, \alpha_{i}^{-1}\right)$ with associated factor sets $\alpha_{i}^{-1}$ such that $\alpha_{i}^{2} \sim 1$.

As mentioned in the introductory section, a set $\operatorname{IrrProj}\left(H_{2}, \alpha_{i}^{-1}\right)$ of 16 irreducible projective characters of $\mathrm{H}_{2}$ with associated factor set $\alpha_{i}^{-1}$ is required in the construction of $\operatorname{Irr}(\bar{G})$, using the technique of Fischer-Clifford matrices. But a choice is to be made amongst the 3 distinct projective characters tables of $\mathrm{H}_{2}$ which contain 16 irreducible projective characters each. The desired set $\operatorname{IrrProj}\left(\mathrm{H}_{2}, \alpha_{i}^{-1}\right)$ of 16 irreducible projective characters can be found in the inertia group $\bar{H}_{2}=2^{4 \cdot} H_{2}$ of $\bar{G}$ on $\operatorname{Irr}(N)$.

Since we have a known permutation representation of $\bar{G}$, the group $\bar{H}_{2}$ is generated within GAP. The normal subgroups $E_{i}$ of order 8 of $\bar{H}_{2}$ are computed. There are three such groups, $E_{1}, E_{2}$, and $E_{3}$, which are elementary abelian. The 3 quotient groups $D_{i}=\bar{H}_{2} / E_{i}$ are double covers for $H_{2}$ with each containing 46 ordinary irreducible characters. 30
TABLE 13: Classes of $\bar{G}$.

\begin{tabular}{|c|c|c|c|c|}
\hline$[g]_{G}$ & $k$ & $f_{j}$ & {$[x]_{\bar{G}}$} & $\left|C_{\bar{G}}(x)\right|$ \\
\hline \multirow{2}{*}{$1 A$} & \multirow{2}{*}{16} & $f_{1}=1$ & $1 A$ & 184320 \\
\hline & & $f_{2}=15$ & $2 A$ & 12288 \\
\hline $2 A$ & 16 & $f_{1}=16$ & $2 B$ & 3840 \\
\hline $2 B$ & 16 & $f_{1}=16$ & $2 C$ & 768 \\
\hline \multirow{3}{*}{$2 C$} & \multirow{2}{*}{16} & $f_{1}=8$ & $4 A$ & 512 \\
\hline & & $f_{2}=8$ & $4 B$ & 512 \\
\hline & \multirow{5}{*}{4} & $f_{1}=1$ & $2 D$ & 768 \\
\hline \multirow{4}{*}{$2 D$} & & $f_{2}=1$ & $4 C$ & 768 \\
\hline & & $f_{3}=1$ & $4 D$ & 768 \\
\hline & & $f_{4}=1$ & $4 E$ & 768 \\
\hline & & $f_{1}=1$ & $2 E$ & 256 \\
\hline \multirow{3}{*}{$2 E$} & \multirow{3}{*}{4} & $f_{2}=1$ & $4 F$ & 256 \\
\hline & & $f_{3}=1$ & $4 G$ & 256 \\
\hline & & $f_{4}=1$ & $4 H$ & 256 \\
\hline \multirow{2}{*}{$3 A$} & \multirow{2}{*}{16} & $f_{1}=1$ & $3 A$ & 2880 \\
\hline & & $f_{2}=15$ & $6 A$ & 192 \\
\hline \multirow{2}{*}{$3 B$} & \multirow{2}{*}{16} & $f_{1}=1$ & $3 B$ & 2880 \\
\hline & & $f_{2}=15$ & $6 B$ & 192 \\
\hline $3 C$ & 1 & $f_{1}=1$ & $3 C$ & 36 \\
\hline $3 D$ & 1 & $f_{1}=1$ & $3 D$ & 36 \\
\hline $3 E$ & 1 & $f_{1}=1$ & $3 E$ & 36 \\
\hline $4 A$ & 4 & $f_{1}=4$ & $8 A$ & 32 \\
\hline $4 B$ & 4 & $f_{1}=4$ & $8 B$ & 32 \\
\hline $5 A$ & 1 & $f_{1}=1$ & $5 A$ & 60 \\
\hline \multirow[t]{2}{*}{$5 B$} & \multirow[t]{2}{*}{1} & $f_{1}=1$ & $5 B$ & 60 \\
\hline & & $f_{1}=1$ & $6 C$ & 48 \\
\hline \multirow{4}{*}{$6 A$} & \multirow{4}{*}{4} & $f_{2}=1$ & $12 A$ & 48 \\
\hline & & $f_{3}=1$ & $12 B$ & 48 \\
\hline & & $f_{4}=1$ & $12 C$ & 48 \\
\hline & & $f_{1}=1$ & $6 D$ & 48 \\
\hline \multirow{3}{*}{$6 B$} & \multirow{3}{*}{4} & $f_{2}=1$ & $12 \mathrm{D}$ & 48 \\
\hline & & $f_{3}=1$ & $12 E$ & 48 \\
\hline & & $f_{4}=1$ & $12 F$ & 48 \\
\hline $6 C$ & 1 & $f_{1}=1$ & $6 E$ & 12 \\
\hline $6 D$ & 1 & $f_{1}=1$ & $6 F$ & 12 \\
\hline $6 E$ & 1 & $f_{1}=1$ & $6 G$ & 12 \\
\hline $10 A$ & 1 & $f_{1}=1$ & $10 A$ & 20 \\
\hline $10 B$ & 1 & $f_{1}=1$ & $10 B$ & 20 \\
\hline $15 A$ & 1 & $f_{1}=1$ & $15 A$ & 15 \\
\hline $15 B$ & 1 & $f_{1}=1$ & $15 B$ & 15 \\
\hline $15 C$ & 1 & $f_{1}=1$ & $15 C$ & 15 \\
\hline $15 D$ & 1 & $f_{1}=1$ & $15 D$ & 15 \\
\hline
\end{tabular}

characters from each of the sets $\operatorname{Irr}\left(D_{i}\right)$ are the liftings of the ordinary irreducible characters of $\mathrm{H}_{2}$ to $\mathrm{D}_{i}$, while the remaining 16 ordinary characters of each $D_{i}$ represent the set $\operatorname{IrrProj}\left(\mathrm{H}_{2}, \alpha_{i}^{-1}\right)$ of irreducible projective characters of $\mathrm{H}_{2}$, which is of interest to us. Using the above GAP code as earlier, the desired set $\operatorname{IrrProj}\left(\mathrm{H}_{2}, \alpha_{i}^{-1}\right)$ of projective characters of $\mathrm{H}_{2}$ which is needed in the construction of the ordinary character table of $\bar{G}$ using Fischer-Clifford theory is identified as Table 3 .

\section{Fischer-Clifford Matrices and Conjugacy Classes of $\bar{G}$}

For the readers who are interested in the construction of the ordinary character table of $\bar{G}=2^{4} \cdot\left(2^{6}:\left(A_{5} \times 3\right)\right)$ using the technique of Fischer-Clifford matrices (see, for example, 
$[6,9,19-23])$, the Fischer-Clifford matrices (Table 12) and the conjugacy classes (Table 13) of $\bar{G}$ are supplied. The conjugacy classes are arranged in a format obtained by the technique of coset analysis (see, for example, $[6,9,21,24]$ ). The set of irreducible characters of $\bar{G}$ will be partitioned into 2 blocks $\Delta_{1}=\left\{\chi_{j} \mid 1 \leq j \leq 26\right\}$ and $\Delta_{2}=\left\{\chi_{j} \mid 27 \leq j \leq\right.$ $42\}$ corresponding to the inertia factor groups $H_{1}$ and $H_{2}$, respectively, where $\chi_{j} \in \operatorname{Irr}(\bar{G})$.

\section{Data Availability}

The data used to support the findings of this study are included within the article.

\section{Disclosure}

The content of this paper was presented as part of the author's short communications' presentation at the 2018 International Congress of Mathematicians in Rio de Janeiro, Brazil.

\section{Conflicts of Interest}

The author declares that there are no conflicts of interest regarding the publication of this paper.

\section{Acknowledgments}

The author would like to thank the financial support from ConfCom and the Faculty of Applied Sciences at Cape Peninsula University of Technology. The author is most grateful to the Lord Jesus Christ.

\section{References}

[1] J. H. Conway, R. T. Curtis, S. P. Norton, R. A. Parker, and R. A. Wilson, Atlas of Finite Groups, Oxford University Press, Oxford, UK, 1985.

[2] R. A. Wilson, P. Walsh, J. Tripp et al., ATLAS of Finite Group Representations, Cambridge University Press, Cambridge, UK, 1998, http://brauer.maths.qmul.ac.uk/Atlas/v3/.

[3] The GAP Group, "GAP-groups, algorithms, and programming, version 4.6.3," 2013, http://www.gap-system.org.

[4] D. Gorenstein, Finite Groups, Harper and Row Publishers, New York, USA, 1968.

[5] B. Fischer, "Clifford-matrices," in Progress in Mathematics, G. O. Michler and C. Ringel, Eds., vol. 95, pp. 1-16, Birkhauser, Basel, Switzerland, 1991.

[6] A. B. M. Basheer and J. Moori, "A survey on Clifford-Fischer theory," London Mathematical Society Lecture Notes Series, vol. 422, pp. 160-172, Cambridge University Press, Cambridge,UK, 2015.

[7] R. J. Haggarty and J. F. Humphreys, "Projective characters of finite groups," Proceedings of the London Mathematical Society, vol. 36, no. 3, pp. 176-192, 1975.

[8] A. L. Prins, "The character table of an involution centralizer in the Dempwolff group $2^{5 \cdot} \mathrm{GL}_{5}(2)$," Quaestiones Mathematicae, vol. 39, no. 4, pp. 561-576, 2016.

[9] F. Ali and J. Moori, "The Fischer-Clifford matrices of a maximal subgroup of $\mathrm{Fi}_{24}$," Representation Theory, vol. 7, pp. 300-321, 2003.
[10] I. M. Isaacs, Character Theory of Finite Groups, Academic Press, San Diego, CS, USA, 1976.

[11] G. Karpilovsky, "Group representations: introduction to group representations and characters,"North-HollandMathematics Studies, vol. 175, North-Holland PublishingCompany, Amsterdam, Netherlands, 1992.

[12] G. Karpilovsky, Projective Representations of Finite Groups, Marcel Dekker, New York, USA, 1985.

[13] E. W. Read, "On the centre of a representation group," Journal of the London Mathematical Society, vol. 16, no. 2, pp. 43-50, 1977.

[14] W. Bosma and J. J. Canon, Handbook of Magma Functions, Department of Mathematics, University of Sydney, Camperdown, Australia, 1994.

[15] A. L. Prins, "The number of irreducible projective characters with associated factor set of any finite group," 2014, https:// mathoverflow.net/users/49528/a-l-prins.

[16] G. Robinson, "The number of irreducible projective characters with associated factor set of any finite group," 2014, https:// mathoverflow.net/users/14450/geoff-robinson.

[17] M. Aschbacher, "Finite group theory," Cambridge Studiesin Advanced Mathematics, vol. 10, Cambrigde University Press, Cambridge, UK, 1986.

[18] J. Schmidt, "Projective characters with corresponding factor set," 2017, https://mathoverflow.net/users/3710/jack-schmidt.

[19] F. Ali and J. Moori, "The Fischer-Clifford matrices and character table of a maximal subgroup of $\mathrm{Fi}_{24}$," Algebra Colloquium, vol. 17, no. 3, pp. 389-414, 2010.

[20] A. Basheer and J. Moori, "Fischer matrices of Dempwolff group $2^{5 \cdot} \mathrm{GL}(5,2)$," International Journal of Group Theory, vol. 1, no. 4, pp. 43-63, 2012.

[21] J. Moori and Z. E. Mpono, "The Fischer-Clifford matrices of the group $2^{6}: \mathrm{SP}_{6}(2), "$ Quaestiones Mathematicae, vol. 22, no. 2, pp. 257-298, 1999.

[22] J. Moori and T. T. Seretlo, "On Fischer-Clifford matrices of a maximal subgroup of the Lyons group Ly," Bulletin of the Iranian Mathematical Society, vol. 39, pp. 1037-1052, 2013.

[23] A. L. Prins and R. L. Monaledi, "Fischer-Clifford matrices and character table of the maximal subgroup of $\left(2^{9}: \mathrm{L}_{3}(4)\right): 2$ of $\mathrm{U}_{6}(2): 2$," International Journal of Mathematics and Mathematical Sciences, vol. 2019, Article ID 9382525, 17 pages, 2019.

[24] J. Moori, "On certain groups associated with the smallest Fischer group," Journal of the London Mathematical Society, vol. 2-23, no. 1, pp. 61-67, 1981. 


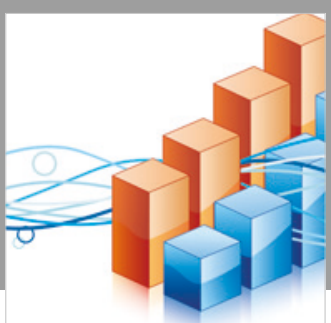

Advances in

Operations Research

\section{-n-m}
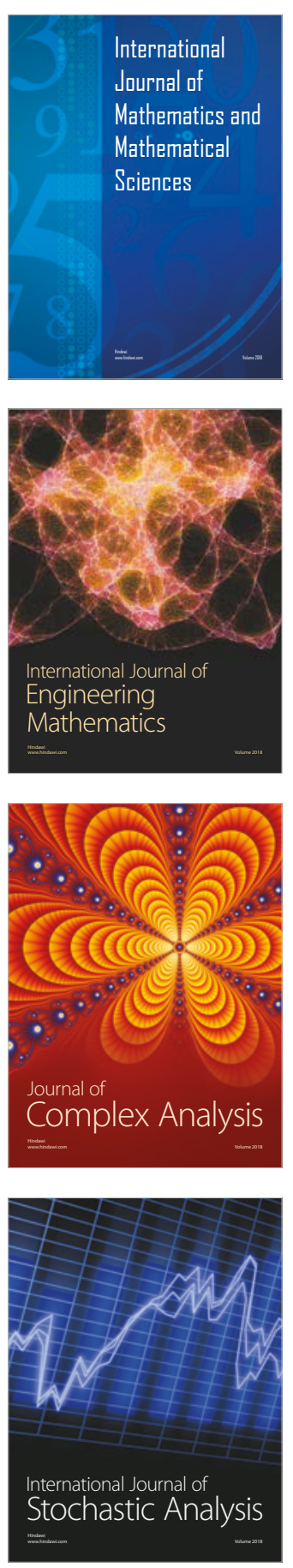
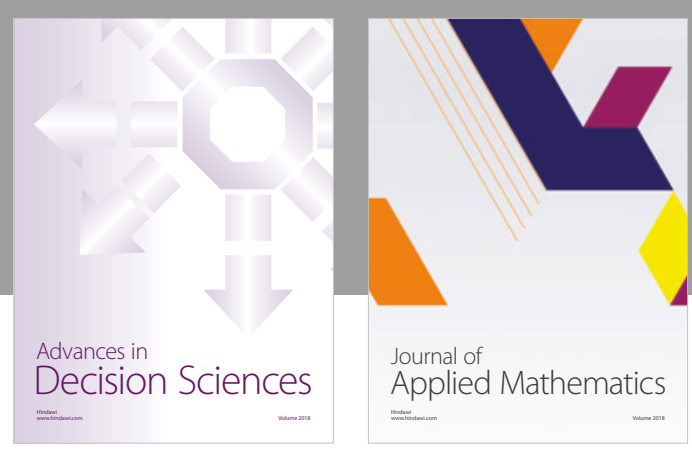

Journal of

Applied Mathematics
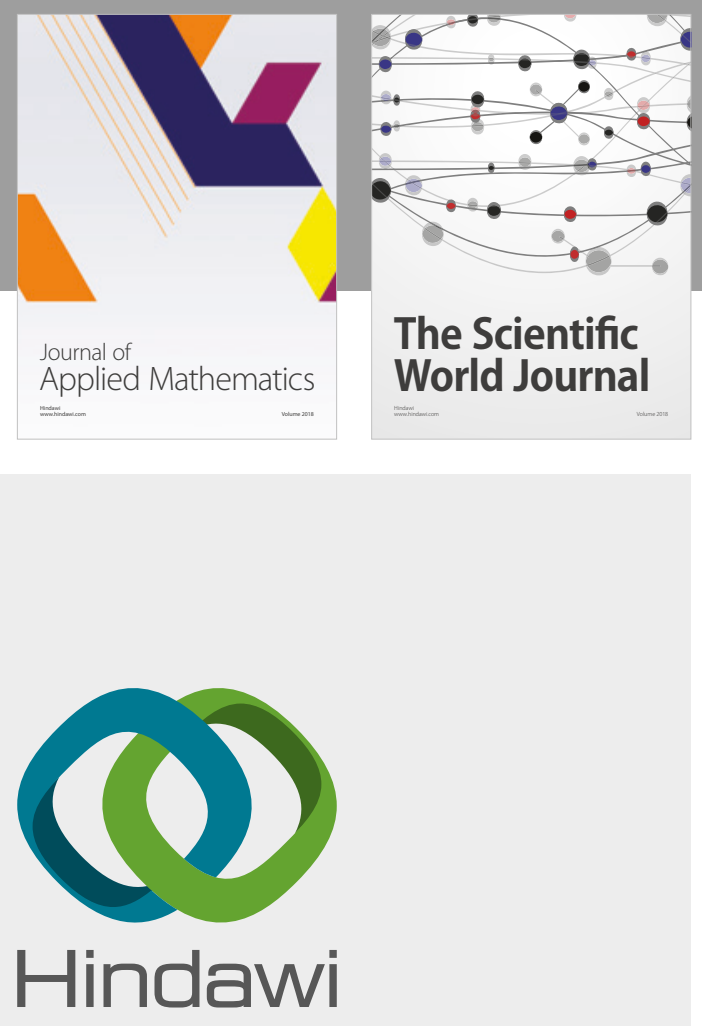

Submit your manuscripts at

www.hindawi.com

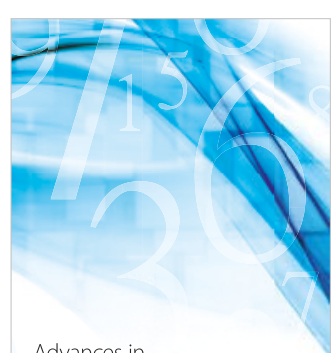

Advances in
Numerical Analysis
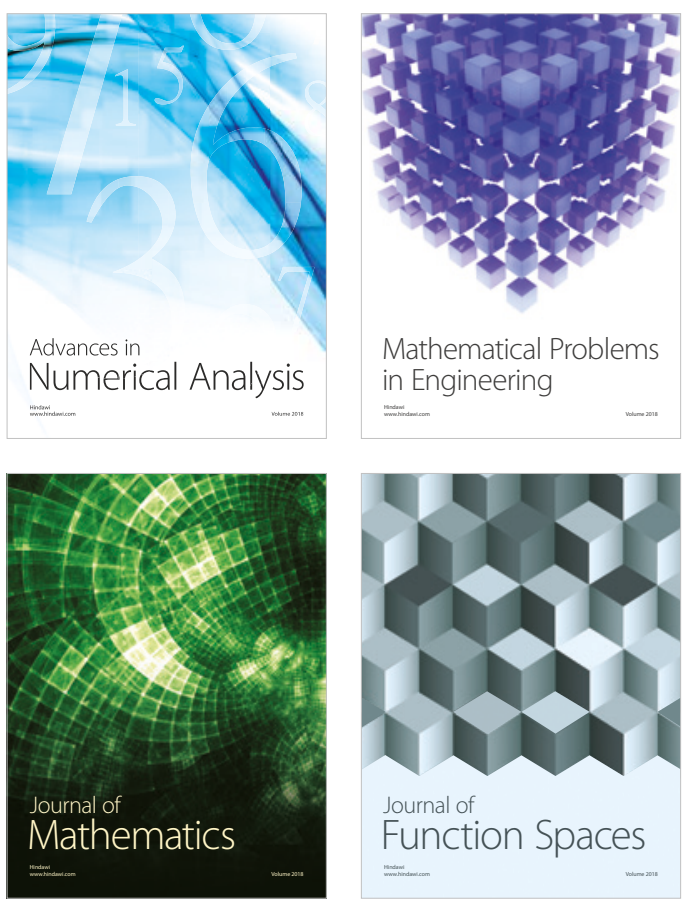

Mathematical Problems in Engineering

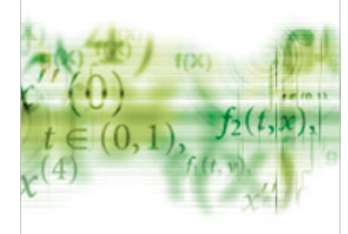

International Journal of

Differential Equations

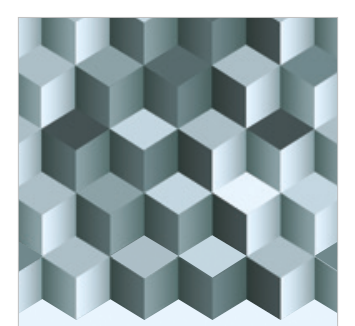

Journal of

Function Spaces

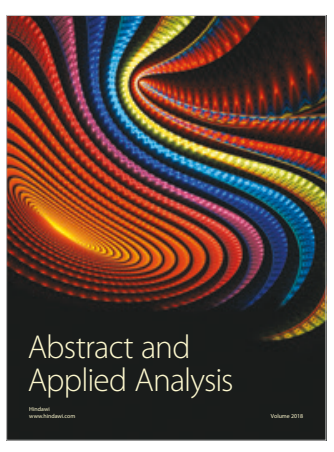

The Scientific

World Journal

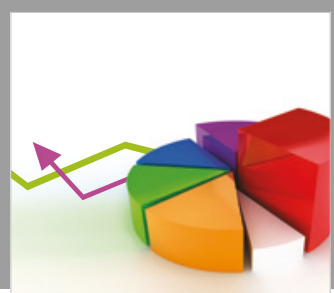

Journal of

Probability and Statistics
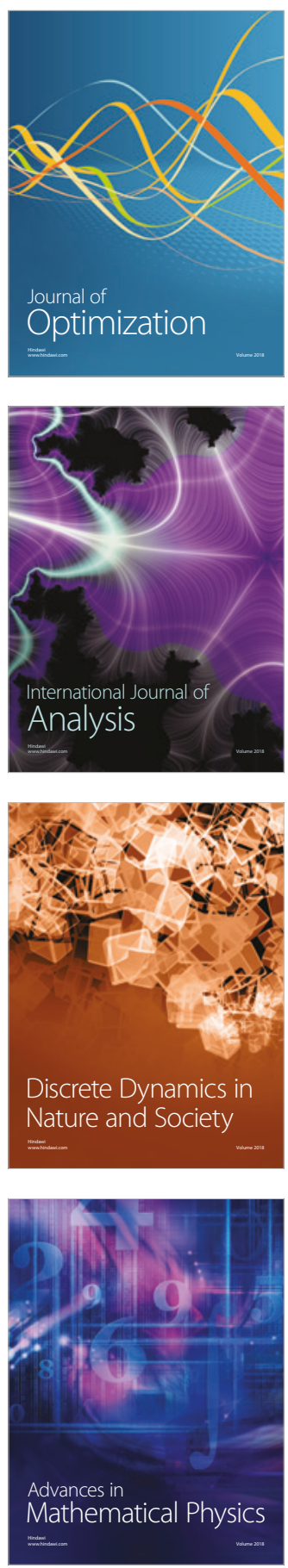